\title{
Preparing the COROT space mission: Incidence and characterisation of pulsation in the lower instability strip ${ }^{\star}$
}

\author{
E. Poretti ${ }^{1}$, R. Garrido 2 , P. J. Amado ${ }^{2}$, K. Uytterhoeven ${ }^{3}$, G. Handler ${ }^{4,5}$, R. Alonso ${ }^{6}$, S. Martín ${ }^{1}$, C. Aerts ${ }^{3}$, \\ C. Catala ${ }^{7}$, M. J. Goupil ${ }^{7}$, E. Michel ${ }^{7}$, L. Mantegazza ${ }^{1}$, P. Mathias ${ }^{8}$, M. L. Pretorius ${ }^{9}$, J. A. Belmonte ${ }^{6}$, A. Claret ${ }^{2}$, \\ E. Rodríguez ${ }^{2}$, J. C. Suarez ${ }^{2,7}$, F. F. Vuthela ${ }^{4,10}$, W. W. Weiss ${ }^{5}$, D. Ballereau ${ }^{11}$, J. C. Bouret ${ }^{12}$, S. Charpinet ${ }^{13}$, \\ T. Hua ${ }^{12}$, T. Lüftinger ${ }^{5}$, N. Nesvacil ${ }^{5}$, and C. Van't Veer-Menneret ${ }^{11}$
}

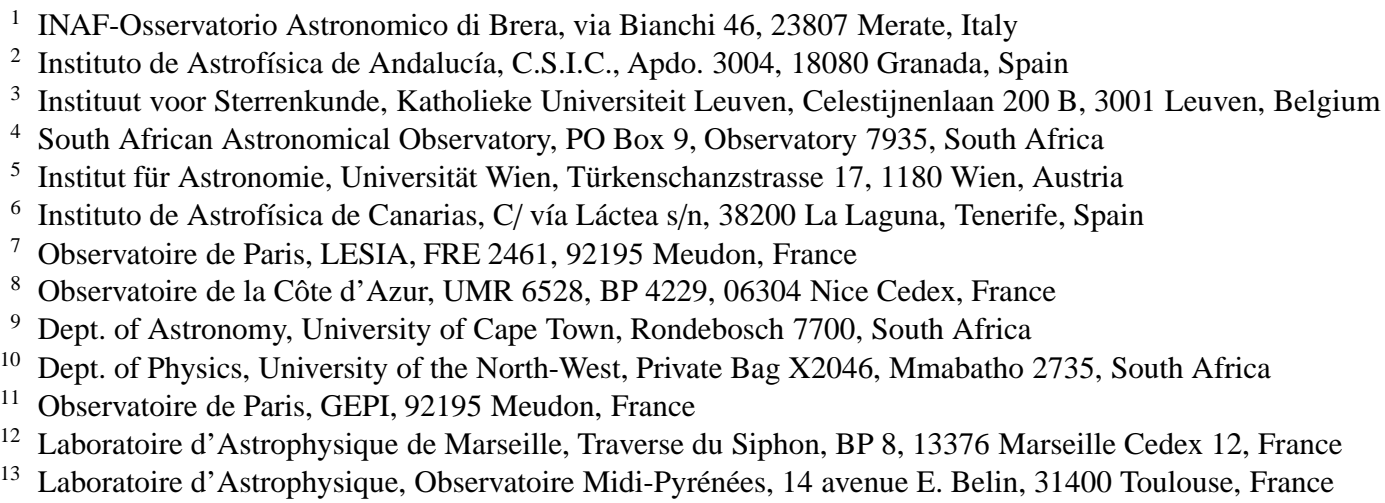

Received 24 February 2003 / Accepted 18 April 2003

\begin{abstract}
By pursuing the goal to find new variables in the COROT field-of-view we characterised a sample of stars located in the lower part of the instability strip. Our sample is composed of stars belonging to the disk population in the solar neighbourhood. We found that $23 \%$ of the stars display multiperiodic light variability up to a few mmag in amplitude, i.e., easily detectable on a single night of photometry. $u v b y \beta$ photometry fixed most of the variables in the middle of the instability strip and high-resolution spectroscopy established that they have $v \sin i>100 \mathrm{~km} \mathrm{~s}^{-1}$. An analysis of the Rodríguez \& Breger (2001) sample ( $\delta$ Sct stars in the whole Galaxy) shows slightly different features, i.e., most $\delta$ Sct stars have a $0.05-\operatorname{mag}$ redder $(b-y)_{0}$ index and lower $v \sin i$ values. Additional investigation in the open cluster NGC 6633 confirms the same incidence of variability, i.e., around $20 \%$. The wide variety of pulsational behaviours of $\delta$ Sct stars (including unusual objects such as a variable beyond the blue edge or a rapidly rotating high-amplitude pulsator) makes them very powerful asteroseismic tools to be used by COROT. Being quite common among bright stars, $\delta$ Sct stars are suitable targets for optical observations from space.
\end{abstract}

Key words. stars: variables: $\delta$ Sct - stars: statistics - stars: oscillations - open clusters and associations: individual: NGC 6633 space vehicles

\section{Introduction}

The preparation of new asteroseismic space missions requires a considerable amount of related theoretical and observational work. In the domain of $\delta$ Sct and related stars the determination of pulsational spectra has so far been performed by intensive, single- or multi-site campaigns, both photometric and spectroscopic. The attention of a wide community of

Send offprint requests to: E. Poretti,

e-mail: poretti@merate.mi.astro.it

* Based on observations collected at the S. Pedro Martír, Sierra Nevada, La Silla, Haute-Provence, South African and Roque de Los Muchachos observatories. specialists has been mostly focused on some well-defined case studies (FG Vir, XX Pyx, 4 CVn, $\theta^{2}$ Tau, BI CMi, 44 Tau; see Poretti 2000 and Breger 2000 for reviews). The scientific plan of the European mission COROT (COnvection, ROtation and planetary Transits; Baglin et al. 2002) has a slightly different strategy, as the satellite will monitor selected targets located in two fields centered at $\alpha=18^{\mathrm{h}} 50^{\mathrm{m}}, \delta=0^{\circ}$ (i.e., in the direction of the Galactic Center) and $\alpha=6^{\mathrm{h}} 50^{\mathrm{m}}, \delta=0^{\circ}$ (Anticenter direction), each having a semi-aperture of $10^{\circ}$. None of the case studies mentioned above is included in these fields.

Consequently suitable candidate target stars have to be searched for. They should be chosen so as to provide a good trade-off between being challenging for aspects of theoretical 
modelling and having suitable observable features. The $\delta$ Sct class covers both the early main-sequence evolutionary stage, when the star is burning hydrogen in the core, and the following one, when it leaves the Terminal-Age Main Sequence (TAMS) burning hydrogen in a shell. These two different stages correspond to different types of structures and their study offers different insights into the physics of the stellar interiors (Breger \& Pamyatnykh 1998). Therefore, both types of variables deserve interest. However, the primary objectives of COROT are highly focused on core overshooting processes and on transport of angular momentum and chemical species. These processes are crucial in the main-sequence stage for intermediate- and high-mass stars. Therefore, we put a strong priority on searching for new $\delta$ Sct stars close to the ZAMS.

Evolved $\delta$ Sct variables pose considerable problems for analysis which limits their use in tests of theory. It is known that evolved models of $\delta$ Sct stars have such a dense frequency spectrum that matching theoretical and observed frequencies might be a hopeless task without further input. As an example, one can consider the prototype of the class, $\delta$ Sct itself, which is included in one of the fields accessible to COROT. Templeton et al. (1997) constructed evolution and pulsation models that match the observed spectral type, luminosity and the identified radial mode frequency of $\delta$ Sct. Accounting for rotational splitting, there are 275 possible $\ell \leq 2$ modes in a $4 \mathrm{~cd}^{-1}$ range (Guzik et al. 2000). Therefore, asteroseismic inferences for such a star will be complicated even for long runs from space.

Rotation is another aspect to keep in mind when evaluating the scientific perspectives of a given target. Fast rotation considerably complicates the modelling of oscillations. By extending the perturbation theory of the influence of rotation on stellar oscillations to the third order (Soufi et al. 1998), asteroseismic inference from oscillations can include relatively high rotation rates. For the mass range of $\delta$ Sct variables this means that stars with equatorial rotational velocities up to $100 \mathrm{~km} \mathrm{~s}^{-1}$ can be modelled, but beyond that the application of perturbation theory remains questionable. Ideally, we would like to scan different rotation rates if we are sure to have reasonably slow rotators among the targets. Finally, stars showing variability at the level of a few mmag (at least) should be preferred as they should guarantee the possibility to complement photometry from space with high-resolution spectroscopy from the ground.

We report here on how we tackled the problem of the identification of suitable targets by means of a theoretical selection followed by an observational one.

\section{Observations and data reduction}

The search for new $\delta$ Sct stars in the COROT field of view has been performed in two observational steps. The first is related to the ground-based activity build-up to determine the physical parameters of all the stars brighter than $V=8.0$ which are included in the COROT accessible fields. This requires that all these stars should be observed at least once in the Strömgren system and at least once with high-resolution spectroscopy. In the Center direction this program was completed well before the summer of 2002. Therefore we know the $u v b y \beta$ indices and the $v \sin i$ values for most of our targets. Photometric observations were carried out at the Sierra Nevada Observatory (automatic six-channel spectrophotometer at the 90-cm telescope), spectroscopic ones at the Haute-Provence Observatory (ELODIE instrument at the 193-cm telescope) and at the La Silla Observatory (FEROS instrument at the 152-cm telescope). The reduction of the photometric data and their transformation into the standard system has been done following the procedures described in Olsen (1993) and references therein. The results of these procedures applied to our dataset will be presented in a future work (Amado et al., in preparation). The $v \sin i$ determinations have been performed taking into account instrumental broadening and limb-darkening effects; uncertainties are of the order of 5-6 $\mathrm{km} \mathrm{s}^{-1}$.

The second observational task is specific to our program. Once the potential targets have been selected, dedicated observing programs were carried out at the Sierra Nevada Observatory, at the S. Pedro Martír Observatory (uvby photometry at the $152-\mathrm{cm}$ telescope - twin photometer of the one at Sierra Nevada), at the South African Astronomical Observatory (50 and 75-cm telescopes with $v$ and $y$ filters) and at the Mercator telescope (120-cm telescope on Canary Islands, Geneva photometric system). Unfortunately, it was not possible to undertake simultaneous two-site campaigns. We decided to monitor 4-5 stars each night, changing the group of stars each night. Of course, such a strategy cannot be considered as totally conclusive about the variability of a specific star. However, our main goal was to scrutinise the whole sample, and to make clear variability detections at the $0.005-$ mag level.

\section{The identification of potential targets}

The $u v b y \beta$ photometry performed at OSN allowed us to construct a colour-magnitude diagram (CMD), which will be one of our main tools to identify the best targets (Fig. 1). We considered some B stars and all the stars belonging to the A and F spectral types; this sample comprises 138 stars. $u v b y \beta$ colour indices were dereddened following the procedure described by Philip et al. (1976); only in $18 \%$ of the cases were the corrections larger than 0.05 mag.

The apparent magnitudes were dereddened using the relation given by Crawford \& Mandwewala (1976). The HIPPARCOS parallaxes were used to determine the absolute magnitude $M_{V}$. In the cases where the HIPPARCos parallaxes were not available, $M_{V}$ values were derived from the Strömgren indices by using standard photometric calibrations.

We also need to define the "lower part of the instability strip". The main sequence is taken from Philip \& Egret (1980) and the $\delta$ Sct instability strip borders from Rodríguez $\&$ Breger (2001). The red border of the $\gamma$ Dor instability strip is taken from Handler \& Shobbrook (2002); the blue border is well inside the $\delta$ Sct instability strip. We also added evolutionary tracks, considering five values for the mass in the range from 1.50 to $2.51 M_{\odot}$. We calculated two sets of models for two different typical overshooting extension distances, 


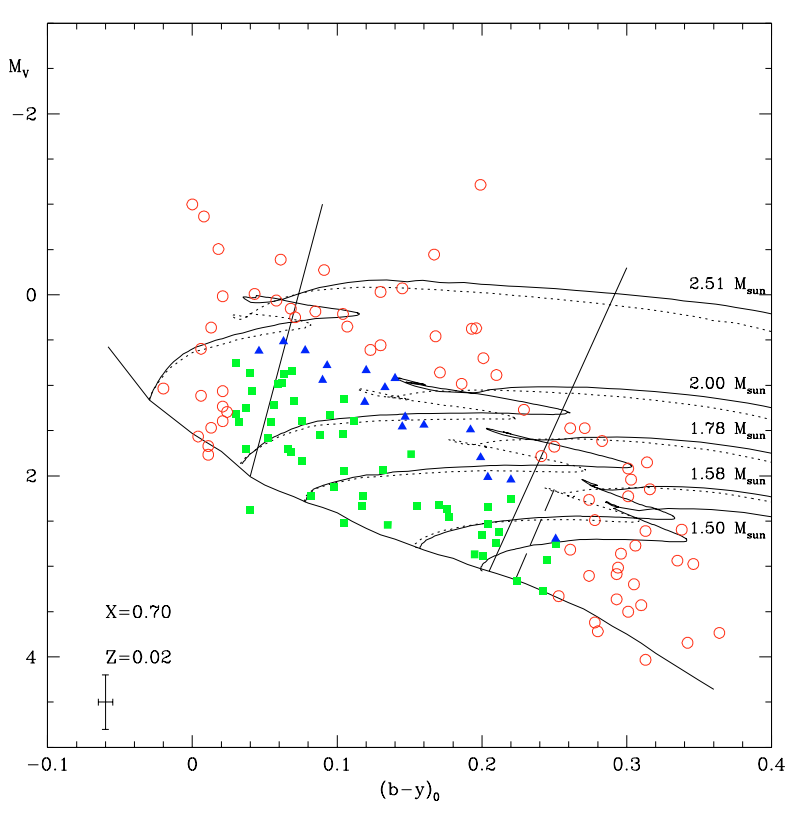

Fig. 1. HIPPARCos unreddened $M_{V}$ against our dereddened $(b-y)_{0}$ colour indices for potential COROT targets in the Center direction. Dotted and solid lines indicate evolutionary tracks for $d_{\text {over }}=0.1$ and $d_{\text {over }}=0.2$, respectively. Solid squares represent stars surely unevolved, independently of overshooting influence. Solid triangles represent stars whose evolutionary status depends on the overshooting importance. Open circles represent stars too advanced on evolutionary tracks or too far outside the instability strip. The borders of the $\delta$ Sct instability strip and the edge of the $\gamma$ Dor domain are also indicated.

i.e., $d_{\text {over }}=0.1$ and $d_{\text {over }}=0.2$ (see Claret 1995 for details). At this point the CMD shown in Fig. 1 is constructed.

Our initial sample consisted of 138 stars; 70 stars out of 138 fall outside the region of interest, i.e., they are too far from the red and blue borders of the instability strip or too advanced on the evolutionary tracks of models with $d_{\text {over }}=0.2$. However, even considering all the stars below the zigzags of $d_{\text {over }}=0.2$ tracks, several of them could actually be evolved stars, depending on the model of choice, with or without overshooting.

To avoid a bias originating from the theory, we divided the stars on the basis of the two different models. Stars located close to the ZAMS are considered unevolved objects and are therefore high-priority targets for the COROT preparation program. On the other hand, the stars approaching the zigzags of $d_{\text {over }}=0.2$ tracks could be evolved ones if the overshooting is not as effective as supposed. These stars are low-priority objects for the COROT program. As can be seen in Fig. 1, limits have been a little relaxed to take into account possible uncertainties in the data, in the dereddening relation, in the calibrations, in the border definitions and so on. Overall average errors of the data and the hydrogen and metal contents of the models are shown in the lower left corner.

Among the 68 selected stars, three variables are known already: HD 183324 (a $\lambda$ Boo star; Paunzen et al. 2002), HD 167858 and HD 175537 (two $\gamma$ Dor stars; Handler 1999). Moreover, HD 182475 and HD 177702 were good candidates to be $\delta$ Sct variables on the basis of previous surveys
Table 1. New and known variable stars located in the COROT accessible field, Center direction.

\begin{tabular}{|c|c|c|c|c|}
\hline Star & $\bar{V}$ & Sp. & $\begin{array}{c}v \sin i \\
{\left[\mathrm{~km} \mathrm{~s}^{-1}\right]}\end{array}$ & $\begin{array}{c}v \text { ampl. } \\
\text { [mmag] }\end{array}$ \\
\hline \multicolumn{5}{|c|}{ New unevolved $\delta$ Sct stars } \\
\hline HD 170699 & 6.95 & A3 & $>200$ & 30 \\
\hline HD 170782 & 7.81 & A2 & 198 & 6 \\
\hline HD 171234 & 7.91 & A4 & 162 & 7 \\
\hline HD 174966 & 7.70 & A3 & 125 & 40 \\
\hline HD 176112 & 7.98 & F0 & 117 & 30 \\
\hline HD 181555 & 7.98 & A5 & 170 & 30 \\
\hline \multicolumn{5}{|c|}{ New evolved $\delta$ Sct stars } \\
\hline HD 174532 & 6.91 & A2 & 32 & 25 \\
\hline HD 177064 & 7.74 & A2 & 180 & 50 \\
\hline HD 177702 & 7.30 & F0 & $>200$ & 150 \\
\hline HD 182475 & 6.61 & A9V & 139 & 35 \\
\hline \multicolumn{5}{|c|}{ Known unevolved $\delta$ Sct star } \\
\hline HD 183324 & 5.79 & $\mathrm{~A} 0 \mathrm{~V}$ & 98 & \\
\hline \multicolumn{5}{|c|}{ Known $\gamma$ Dor variables } \\
\hline HD 167858 & 6.62 & $\mathrm{~F} 2 \mathrm{~V}$ & 10 & \\
\hline HD 175337 & 7.39 & F5 & 39 & \\
\hline HD 169577 & 8.64 & F0 & & \\
\hline \multicolumn{5}{|c|}{ New very evolved $\delta$ Sct star } \\
\hline HD 172588 & 7.22 & FOII-III & 12 & \\
\hline \multicolumn{5}{|c|}{ Known very evolved $\delta$ Sct stars } \\
\hline$\delta$ Sct & 4.70 & F2IIIp & 27 & \\
\hline HD 174553 & 9.35 & F8 & 42 & 130 \\
\hline \multicolumn{5}{|c|}{ Suspected $\gamma$ Dor star } \\
\hline HD 178596 & 5.24 & FOIII-IV & 77 & 15 \\
\hline \multicolumn{5}{|c|}{ Geometrical variables? } \\
\hline HD 171802 & 5.37 & F5III & 13 & 40 \\
\hline HD 172506 & 7.96 & $\mathrm{~F} 2$ & 47 & 20 \\
\hline HD 179123 & 7.40 & A5 & 10 & 10 \\
\hline
\end{tabular}

(Hildebrandt 1992 and Handler 2002, respectively): we kept them in our sample for confirmation. Therefore, we have 65 stars that are good theoretical candidate $\gamma$ Dor or $\delta$ Sct pulsators.

\section{The detection of variability}

Despite the large number of stars to be observed and the accuracy required in the measurements, only a few stars could not be evaluated. Four stars have a close companion: they cannot be measured as single stars neither from the ground, nor from space, as the defocused images of two close stars will result in inaccurate photometry. Another star is too bright to be measured by COROT. For one star we could obtain only a few measurements suggesting constant brightness.

Therefore, we successfully monitored 59 stars. We discovered at least 13 stars displaying evident light variations and we confirmed the $\delta$ Sct variability of HD 182475 and HD 177702, obtaining more reliable light curves. All these variables are listed in Table 1. 


\subsection{The new $\delta$ Sct stars}

The sample of $\delta$ Sct stars discovered in the Center direction is quite representative of the different types of behaviour known for this class of pulsating star (Fig. 2).

The simplest variables are HD 170782 and HD 171234, which show almost regular light curves. The frequency analysis of the HD 170782 (Fig. 2, curve at top) data yields a main term at $f=25.0 \mathrm{~cd}^{-1}$ and the subsequent least-squares fit a full-amplitude of 6.0 mmag in $v$ light, with a rms residual of 2.8 mmag. In the case of HD 171234 we got a main term at $f=23.6 \mathrm{~cd}^{-1}$, a full-amplitude of $6.8 \mathrm{mmag}$ in $v$ light and a rms residual of $4.0 \mathrm{mmag}$.

The multiperiodic behaviour of HD 177064, HD 170699, HD 174966, HD 182475 and HD 181555 is clearly visible on a single night. For example, during the second half of the light curve of HD 182475, the rapid variability disappears and a long cycle takes place (Fig. 2, second curve from top). Changes in the total amplitude oscillation are seen in HD 170699 , HD 174966 (Fig. 2, third curve from top) and HD 177064 (Fig. 2, fourth curve from top). The photometric variability of HD 174966 is corroborated by the spectroscopic detection of line profile variations. HD 181555 shows other features in its light curve, i.e., oscillations are always visible, but with changing shapes and different time intervals between consecutive extrema.

The light curve of HD 176112 (Fig. 2, fifth curve from top) adds more complications, as it suggests the possibility of a long term variation ( $\gamma$ Dor pulsation or geometrical variability) superposed on a rapid one ( $\delta$ Sct pulsation). Further observations are necessary to characterise this variable star better, also considering that HIPPARCOS photometry is inconclusive in this respect.

To conclude the journey along the different behaviours of our $\delta$ Sct stars, HD 177702 (Fig. 2, curve at bottom) provides a good example of a high amplitude light curve (0.17 mag in $v$ ). High-resolution spectroscopy allowed us to determine $v \sin i>200 \mathrm{~km} \mathrm{~s}^{-1}$, a value quite unusual for a high-amplitude $\delta$ Sct star.

\subsection{Stars showing slow variability}

When observing such a large sample, we should expect some unclear cases, especially measuring each target on one night only. Indeed, we found five of such unclear cases.

The light curves of HD 172506 and HD 171802 show a continuous change in brightness, up to 0.04 mag in $v$ light. The spectral types of both stars are compatible with those of $\gamma$ Dor pulsators. However, the variability is not discernible in the $v-y$ colour curve and therefore geometrical variability due to binarity seems more probable. We also note that the high-resolution spectrogram of HD 172506 does not show two sets of spectral lines or noticeable line profile deformations. Moreover, the analysis of the HIPPARCos photometry did not show any evidence for variability: the amplitude spectra have peak noise levels of 3 mmag for HD 171802 and 8 mmag for HD 172506. In the case of HD 179123 the $v$-amplitude is only

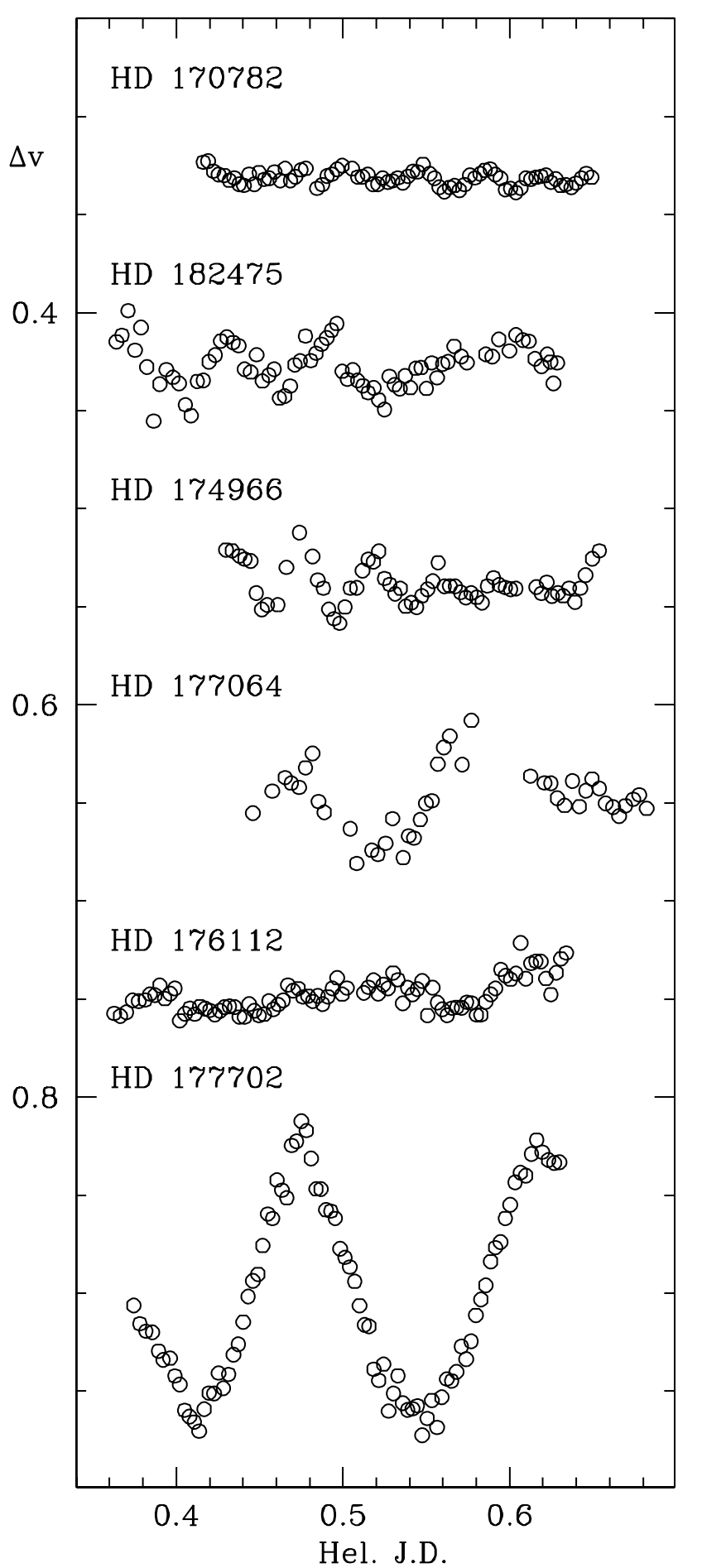

Fig. 2. Light curves of some $\delta$ Sct discovered in the field accessible to COROT.

$0.01 \mathrm{mag}$. The star can be a $\gamma$ Dor variable $\left((b-y)_{0}=0.212\right)$, but a rotational or even a spurious effect are also plausible.

Therefore, the variability of HD 172506, HD 171802 and HD 179123 needs further confirmation; the three stars are considered non-pulsating stars (reported as "Geometrical variables?" in Table 1) in the following discussion.

On the other hand, the drift in the HD 178596 data is visible both in the $v$ and in the $v-y$ curves. Taking also into account that the high-resolution spectrograms show line profile 
deformations and that the star is located close to the red border of the instability strip, the $\gamma$ Dor hypothesis seems preferable to a geometrical variability (the star is reported as "Suspected $\gamma$ Dor" in Table 1). However, we do not include it in the list of pulsating variables, waiting for further confirmation; we note that the HIPPARCOS photometry is also inconclusive.

Finally, for one star (HD 173611) the light curve shows a scatter which did not allow us to select between variability or constancy.

\subsection{Constant stars}

Forty-four stars did not display detectable light variation. Of course, they can be variables on longer time scales or can have very small amplitude, not detectable in our short single-night runs. In the former case they would not be of particular interest, in the latter case the observational effort to detect variability would require a long and intensive survey which we cannot undertake at this stage for such a large sample. We note that some photometrically constant stars actually show spectroscopic variability. This is not surprising, as it is well known that high-degree nonradial pulsation modes can produce cancellation effects in photometry, but can still be detectable by spectroscopy.

The quality of a given night biases the variability detection. As an example, the standard deviations of the time series for HD 185090, HD 170274 and HD 180086 (obtained on the nights of JD 2452488 and 2452489) and on HD 176921 and HD 177011 (obtained on the night of JD 2452467) are well above $6.5 \mathrm{mmag}$, suggesting non-perfect photometric nights. In this context, we stress that the strategy to observe each group of stars just on one night was the only viable one, taking into account the large number of stars to be monitored and the limited telescope time available.

\section{Impact of the results on general scientific topics and on specific COROT aspects}

The results described above will be used to characterize the potential COROT targets and to build the observational program. Besides that, they can be used to investigate pulsation in the lower instability strip.

\subsection{The variability in the lower part of the instability strip}

Figure 3 shows the positions of already known and new $\delta$ Sct stars located in the lower part of the instability strip. For sake of homogeneity, we considered only well-established variable and constant stars, i.e., the unclear cases discussed in Sect. 4.2 are omitted. The magnitude limit $V \leq 8.0$ implies that we observed as far as $310 \mathrm{pc}$ in a cone with a full aperture of $24^{\circ}$ (galactic latitudes $b^{\mathrm{II}}$ between $-12^{\circ}$ and $+12^{\circ}$ ) in the direction of the galactic centre (galactic longitudes $l^{\mathrm{II}}$ between $+22^{\circ}$ and $+45^{\circ}$ ), which in turn results in a maximum distance of $70 \mathrm{pc}$ from the galactic plane.

We arbitrarily divided the lower part of the instability strip in 7 boxes, with margins roughly parallel to the borders.

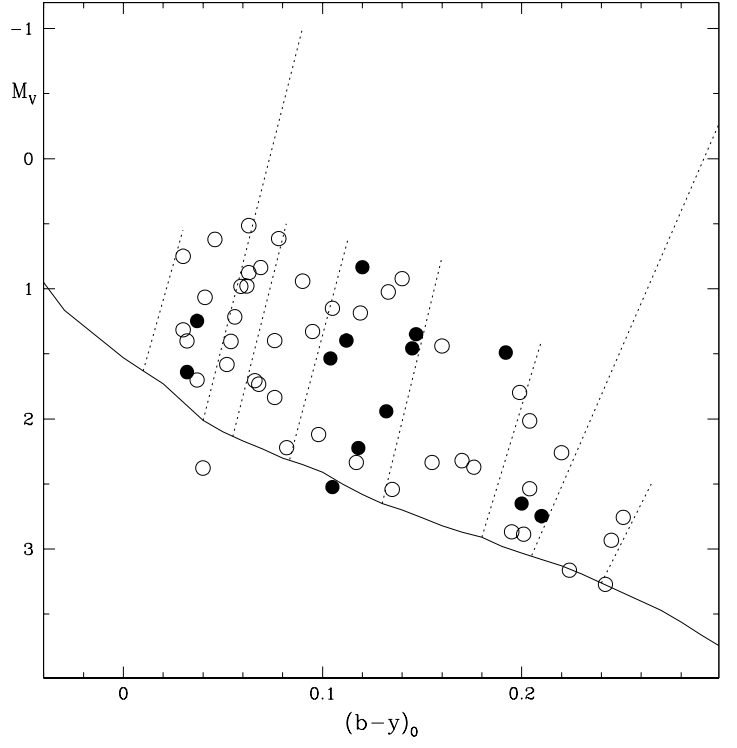

Fig. 3. Incidence of variability in our sample. The boxes are selected taking the borders roughly parallel to the blue and red borders (the longest ones) of the instability strip. Filled circles: variable stars. Open circles: constant stars.

The resulting histogram is shown in Fig. 4. Looking at Figs. 3 and 4 , we note that:

1. the variable stars are concentrated in the central box;

2. two stars, HD 170782 and the $\lambda$ Boo variable HD 188324, are outside the instability strip on the blue edge;

3. the two variables close to the red border are $\gamma$ Dor stars;

4. only one $\delta$ Sct star, HD 181555, is located near the ZAMS. Most stars are more than 0.5 mag above it.

Breger (2000) reports the incidence of variability in the lower part of the instability strip by analysing stars spread all over the sky. The incidence is around 50\% in the central zone; when considering the whole instability strip the percentage decreases to around $20 \%$. In our sample we have 13 variables out of 57 stars $(23 \%)$. In the central box we have 8 variables out of $14(57 \%)$. Of course, the percentages change as a function of the threshold of variability. Both our and Breger's samples are limited to stars having an amplitude larger than 3-4 mmag: therefore, it is no surprise to find the same percentages.

However, the figures we obtained suggest that the incidence of appreciable $\delta$ Sct photometric variability (i.e., the excitation of modes with $\ell \leq 3$ resulting in light changes up to the mmag level and more) is a function of the position in the CMD. To obtain more input on this point, we analysed the sample provided by Rodríguez \& Breger (2001; R00 sample). We considered the 210 low-amplitude $\delta$ Sct stars located in the same part of the instability strip: we thus handled a group of stars similar to ours, except for the space distribution. We obtained the histogram shown in Fig. 5, where variability is well represented in the central box and is also more common toward the red edge. As can be derived from the magnitude limit and galactic coordinates reported above, the COROT sample is representative of the disk population located in the solar neighbourhood. Comparing Figs. 4 and 5, the COROT sample appears slightly 


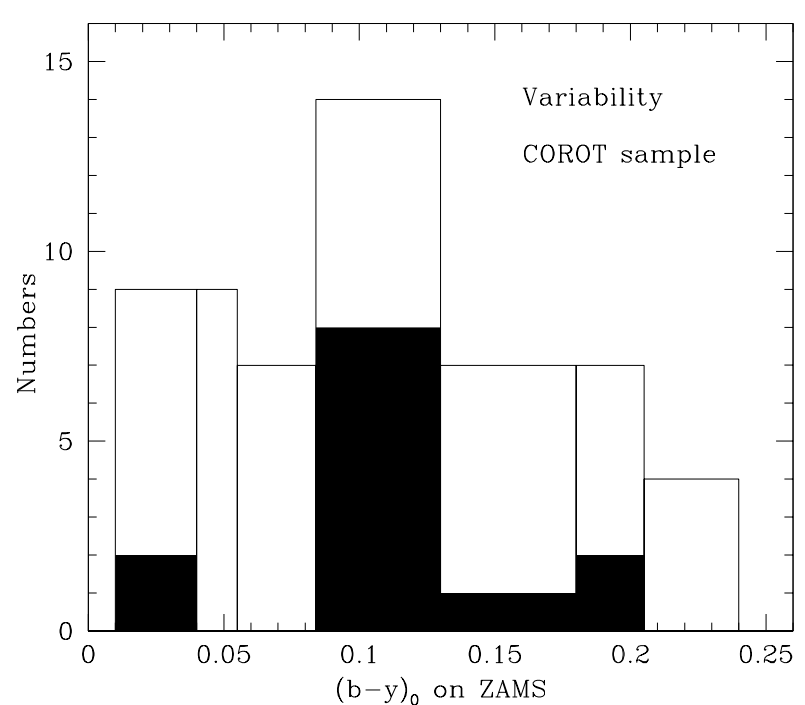

Fig. 4. Histograms of stars in the photometric boxes selected in Fig. 3. Note that the $(b-y)_{0}$ values are taken on the ZAMS. The black areas indicate the number of pulsating stars, i.e., both $\delta$ Sct and $\gamma$ Dor variables.

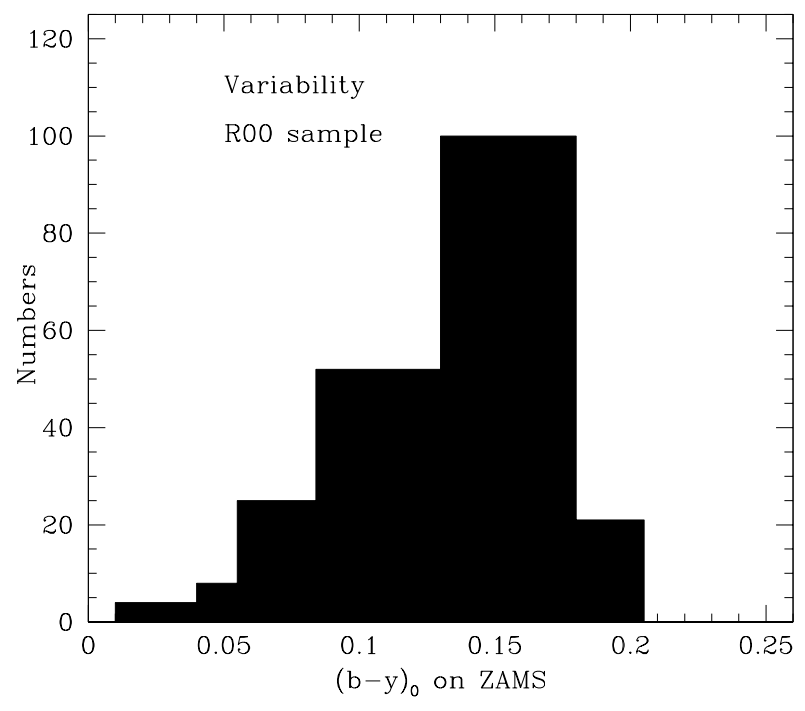

Fig. 5. Histograms of $\delta$ Sct variables in the same photometric boxes considered in Fig. 3, but considering the R00 sample (Rodríguez \& Breger 2001).

bluer than the R00 sample. If real, such a difference can originate only in the specific environment where COROT stars are located; it is diluted when the sample decreases in homogeneity, i.e., when it is extended to the whole Galaxy. Therefore, the particular chemical composition of solar neighbourhood seems to enhance variability in hot stars. Selection effects are also plausible. Indeed, even if the photometric accuracy of our data is quite good, our limited time sampling can miss the $\delta$ Sct stars located close to the red border, where the amplitudes are smaller.

We also note that HD 170782 re-opens the problem of variables beyond the blue border of the instability strip. Rodríguez \& Breger (2001) found a few candidates, but they suggest that the variability was proposed on the basis of inaccurate photometry. In the case of HD 170782 the light variability is quite

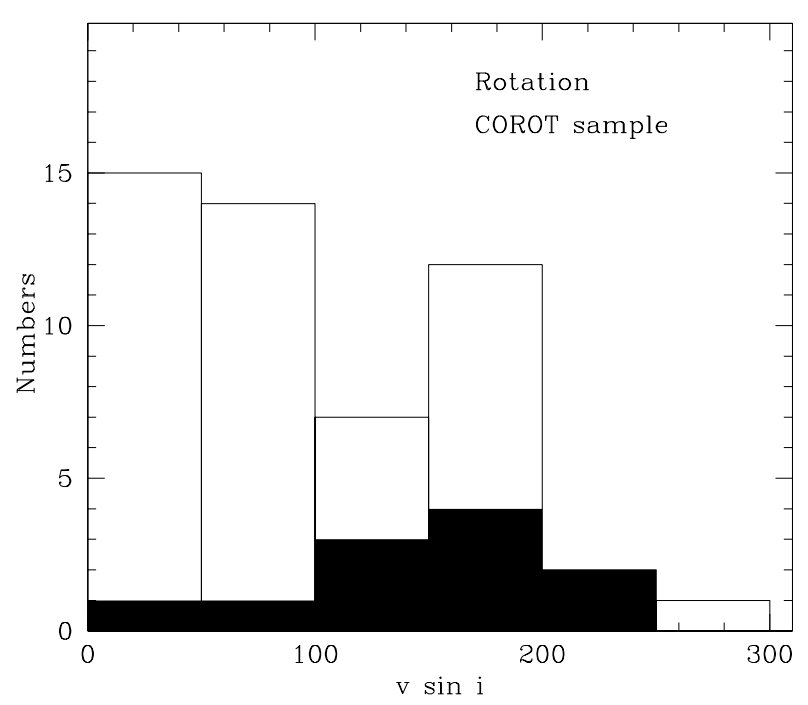

Fig. 6. Histograms of $\delta$ Sct variables (black area) and constant stars (white area) as function of the measured $v \sin i$.

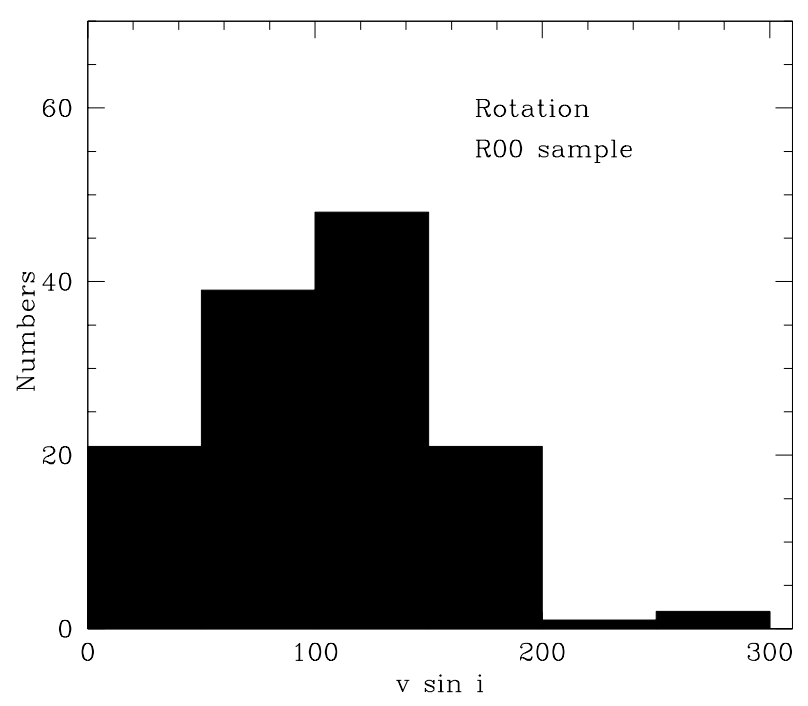

Fig. 7. Histograms of the $v \sin i$ values of $\delta$ Sct variables in the R00 sample (Rodríguez \& Breger 2001).

evident (Fig. 2) and the position in the CMD too far from the blue border to be ascribed to uncertainties in the $(b-y)_{0}$ photometry. Pamyatnykh (2000) demonstrated that $\delta$ Sct pulsation should not occur for these stars in the classical picture. To find a solution, all these stars should be studied individually. For example, some of them may have higher helium abundance (in which case the blue edge will be hotter), for some of them standard photometric calibrations may not apply owing to chemical peculiarities, or some other parameter may play a subtle rôle.

\subsection{The rotational velocity of photometrically variable stars}

There is a well-established relationship between photometric amplitude and $v \sin i$ values, i.e., high-amplitude pulsators are slow rotators (see Fig. 5 in Breger 2000). For amplitudes larger than $0.10 \mathrm{mag}, v \sin i$ is below $50 \mathrm{~km} \mathrm{~s}^{-1}$. In our sample there is the remarkable exception of HD 177702 (see Fig. 2, curve at 


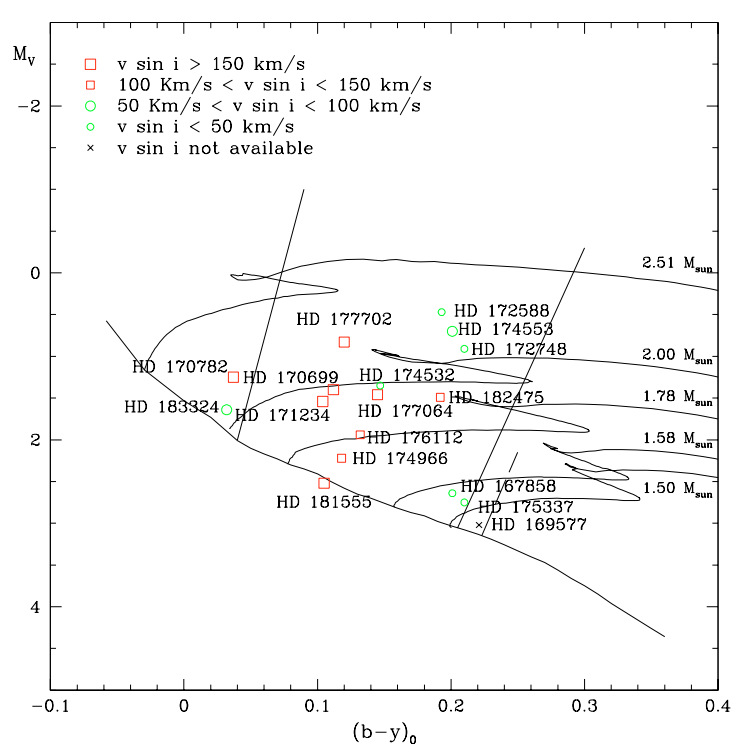

Fig. 8. Hipparcos unreddened absolute visual magnitudes $M_{V}$ against dereddened $(b-y)_{0}$ data for stars of interest in the Center direction. The theoretical tracks with $d_{\text {over }}=0.2$ are shown. All the variables are $\delta$ Sct stars, with the exception of the $\gamma$ Dor stars HD 169577, HD 167858 and HD 175337.

bottom), which shows a $v$ amplitude of $0.17 \mathrm{mag}$, which scales to about $0.12 \mathrm{mag}$ in $V$ light, and $v \sin i>200 \mathrm{~km} \mathrm{~s}^{-1}$. The star is far from the ZAMS and its combination of high amplitude, fast rotation and evolved evolutionary state is unusual amongst the $\delta$ Sct stars. Further investigations are necessary to characterise HD 177702 both from a theoretical (evolution and rotation) and observational (high amplitude as a result of multiperiodicity?) point of view.

We also investigated the effect of rotation on variability. We remind the reader that the break-up velocity is around $250 \mathrm{~km} \mathrm{~s}^{-1}$ for masses and radii typical of $\delta$ Sct variables. Considering the $11 \delta$ Sct stars, 6 objects have $v \sin i>$ $150 \mathrm{~km} \mathrm{~s}^{-1}$, and 9 stars have $v \sin i>100 \mathrm{~km} \mathrm{~s}^{-1}$. Moreover, when considering all the stars having $v \sin i>150 \mathrm{~km} \mathrm{~s}^{-1}$, 9 are found to be photometrically constant, 6 are found to be variable. Figure 6 summarises what we found in our sample: it appears that light variability is concentrated in the 100$200 \mathrm{~km} \mathrm{~s}^{-1}$ range. When considering the R00 sample, the histogram of the available $v \sin i$ values (132 stars out of 210 previously selected) shows the highest peak in the 100-150 $\mathrm{km} \mathrm{s}^{-1}$ range (Fig. 7). The strong dependence on an unknown parameter such as the inclination angle $i$ smoothes the differences between the two samples, preventing physical inferences. We can just suggest that a rotational velocity of $100-150 \mathrm{~km} \mathrm{~s}^{-1}$ is quite common among $\delta$ Sct stars.

\subsection{Our results in the COROT framework}

By combining the results from Figs. 1 and 3 we obtain Fig. 8. The evolved $\delta$ Sct stars HD 172748 $\equiv \delta$ Sct, HD 172588, HD 174553 and the faint $\gamma$ Dor variable HD $169577(V=8.64$; see Sect. 6) are added for completeness. This figure illustrates the scientific output of the action undertaken to identify new potential COROT targets in the Center direction. We note that

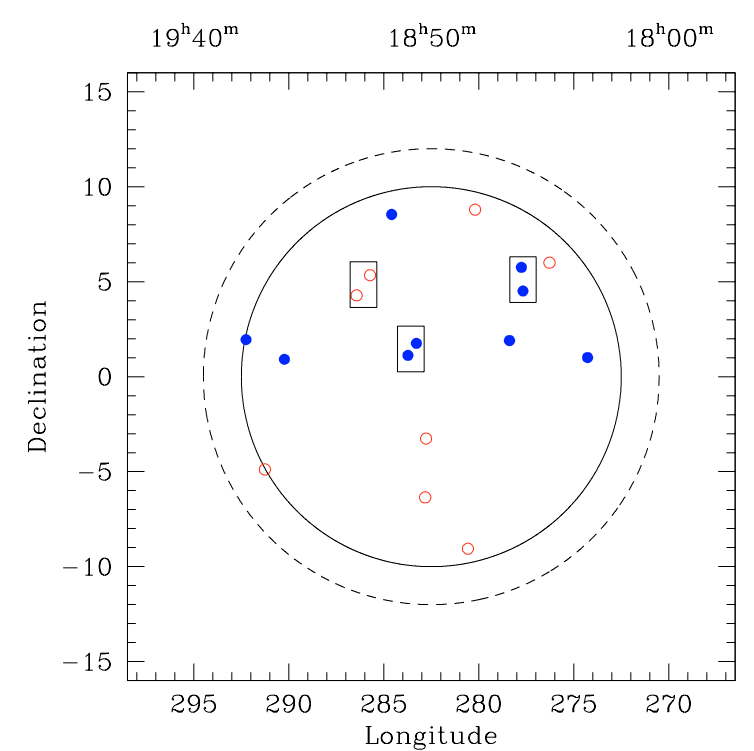

Fig. 9. The location of the stars of interest in the COROT accessible field (solid and dashed lines, indicating different levels of stray light contamination). Filled circles are high-priority stars, open circles are low-priority ones. Three pairs of stars can be observed together in the Seismo CCD (whose field-of-view is indicated by the small rectangles).

by observing a zone of $450 \mathrm{deg}^{2}$ on or just above the galactic plane we discovered variables well mapping the lower part of the instability strip.

Figure 9 shows the positions of the stars of interest in the COROT field-of-view. The solid circle indicates the $10^{\circ}$ cone, where COROT will supply best results. The observing cone can be extended up to $12^{\circ}$ (dashed line) for objects of special interest; above that limit stray light would cause a strong degradation in the photometric accuracy. Three pairs of stars are observable in the same field of view of the Seismology CCD: HD 170782-HD 170699 (right), HD 174966-HD 175337 (middle) and HD 177702-HD 177064 (left).

HD 174532 is the only $\delta$ Sct star showing a low $v \sin i$ value $\left(32 \mathrm{~km} \mathrm{~s}^{-1}\right)$. None of the $\delta$ Sct stars close to the ZAMS has a small $v \sin i$ (the lower limit is the $90 \mathrm{~km} \mathrm{~s}^{-1}$ observed for the $\lambda$ Boo variable HD 183324). The search for smaller amplitude variability (less than $3 \mathrm{mmag}$ ) in slow rotators close to the ZAMS (see Table 2 for suitable targets) will be the goal of future dedicated observations; it should also allow us to improve the percentages discussed above, as we will be able to infer how many variables we missed in the previous search.

\section{The open cluster NGC 6633}

The open cluster NGC 6633 is also located in the field accessible to COROT. A multisite campaign (Martín \& Alonso, in preparation) has been planned to search for variable stars: indeed, the cluster could be a suitable target for COROT. The turn-off point is just above the blue edge. With a distance modulus $m-M=7.77$, the members located in the lower part of the instability strip are too faint $(9.5<V<11.2)$ to be COROT primary targets, but they can provide a further test for the incidence of variability in a well-defined environment. 
Table 2. Stars not showing an evident trace of variability. They are considered as constant for our purposes. $N$ is the number of measurements; $s . d$. is the standard deviation. The note "_" indicates stars used once as comparison stars and not re-observed.

\begin{tabular}{|c|c|c|c|c|c|}
\hline Star & $\bar{V}$ & Sp. & $\begin{array}{c}v \sin i \\
{\left[\mathrm{~km} \mathrm{~s}^{-1}\right]}\end{array}$ & $\bar{N}$ & $\begin{array}{c}\text { s.d. } \\
{[\mathrm{mmag}]}\end{array}$ \\
\hline \multicolumn{6}{|l|}{ Unevolved stars } \\
\hline HD 166991 & 6.83 & $\mathrm{~A} 2$ & 184 & & - \\
\hline HD 167946 & 7.34 & $\mathrm{~A} 0$ & 53 & 76 & 3.2 \\
\hline HD 167968 & 7.75 & $\mathrm{~A} 2$ & 199 & 58 & 3.1 \\
\hline HD 169268 & 6.36 & F6 & 20 & 52 & 3.9 \\
\hline HD 169436 & 7.71 & $\mathrm{~F} 2$ & 162 & 59 & 3.8 \\
\hline HD 170818 & 7.24 & $\mathrm{~F} 2$ & 86 & 79 & 3.5 \\
\hline HD 171149 & 6.35 & $\mathrm{~A} 0 \mathrm{Vn}$ & 288 & 62 & 3.2 \\
\hline HD 171834 & 5.44 & F3V & 72 & 69 & 2.8 \\
\hline HD 171836 & 7.70 & F0 & 60 & & - \\
\hline HD 173073 & 7.67 & A0 & 66 & & - \\
\hline HD 173369 & 7.99 & $\mathrm{~A} 2$ & 23 & 90 & 5.2 \\
\hline HD 174162 & 7.76 & $\mathrm{~A} 0$ & 156 & 58 & 5.9 \\
\hline HD 175272 & 7.44 & F5 & 23 & & - \\
\hline HD 175543 & 7.06 & $\mathrm{~A} 5 \mathrm{~V}$ & 12 & 59 & 4.5 \\
\hline HD 176921 & 8.00 & A2 & $<10$ & 25 & 8.5 \\
\hline HD 177011 & 7.20 & $\mathrm{~A} 0$ & $<10$ & 25 & 7.3 \\
\hline HD 177177 & 7.82 & $\mathrm{~A} 2$ & $<10$ & 45 & 6.4 \\
\hline HD 177178 & 5.83 & $\mathrm{~A} 4 \mathrm{~V}$ & 176 & 27 & 2.7 \\
\hline HD 177332 & 6.72 & $\mathrm{~A} 5 \mathrm{~m}$ & 90 & & - \\
\hline HD 177552 & 6.54 & F1V & 41 & 50 & 3.7 \\
\hline HD 177959 & 7.28 & A3 & 136 & 61 & 3.8 \\
\hline HD 178190 & 7.11 & A2 & 151 & 57 & 2.7 \\
\hline HD 178265 & 7.19 & F0 & 54 & & - \\
\hline HD 178409 & 7.90 & $\mathrm{~A} 0$ & 54 & 67 & 4.1 \\
\hline HD 178857 & 7.72 & $\mathrm{~A} 0$ & 58 & 25 & 2.4 \\
\hline HD 178954 & 6.83 & A0 & 144 & & - \\
\hline HD 179739 & 7.90 & $\mathrm{~A} 2$ & & 31 & 7.0 \\
\hline HD 179742 & 7.66 & $\mathrm{~F} 1$ & $<10$ & 25 & 2.6 \\
\hline HD 179892 & 7.82 & $\mathrm{Am}$ & 90 & 49 & 5.5 \\
\hline HD 179939 & 7.22 & A3 & 52 & & - \\
\hline HD 181414 & 7.07 & $\mathrm{~A} 2$ & 8 & & - \\
\hline HD 182623 & 7.82 & $\mathrm{~A} 0$ & $<10$ & & - \\
\hline HD 183265 & 7.33 & A0 & & 50 & 4.6 \\
\hline HD 185090 & 7.31 & A5 & & 72 & 6.7 \\
\hline \multicolumn{6}{|l|}{ Evolved stars } \\
\hline HD 169310 & 7.52 & A3 & 134 & 52 & 5.7 \\
\hline HD 169725 & 6.85 & A3 & 16 & 52 & 4.2 \\
\hline HD 170274 & 7.86 & $\mathrm{~F} 1$ & 31 & 65 & 8.3 \\
\hline HD 174589 & 6.08 & F2III & 97 & & - \\
\hline HD 174866 & 6.37 & $\mathrm{~A} 7 \mathrm{Vn}$ & 184 & 67 & 5.3 \\
\hline HD 175015 & 7.81 & $\mathrm{~A} 0$ & 135 & & - \\
\hline HD 175250 & 7.05 & B9 & & & - \\
\hline HD 175664 & 7.75 & A0 & 51 & 63 & 5.1 \\
\hline HD 176074 & 7.09 & $\mathrm{~A} 2$ & 18 & & - \\
\hline HD 180086 & 6.63 & F0 & 197 & 72 & 6.8 \\
\hline
\end{tabular}

We established the membership by using the BDA database developed by Mermilliod (1995), Sanders (1973) and Hiltner et al. (1958). A preliminary analysis allowed us to firmly decide on the variability of six (five $\delta$ Sct stars and one $\gamma$ Dor star) out of 30 members. In the case of two $\delta$ Sct stars the membership is not conclusive; moreover, the available photometry on additional six members does not allow us to choose between variability or constancy. Therefore, we can propose a value of $20 \%$ (6 out of 30) for the incidence of variability, with small fluctuations owing to suspected stars and uncertain memberships. Such a result substantially confirms the value obtained for isolated stars.

Cluster variables of other classes also have been found (among them, two Ap stars and one eclipsing binary brighter than $V=9.5$ ). Other pulsating stars are not included in the above statistic as they are considered non-members: the previously-known $\gamma$ Dor star HD $169577(V=8.64)$ (Martín $\&$ Rodríguez 2002), the two new multiperiodic $\delta$ Sct stars HD $169597(V=9.13)$ and $\mathrm{BD}+06^{\circ} 3737(V=9.15)$. Therefore, in Fig. 9, the open circle close to the pair at the right marks not only the position of HD 169577 but also the position of all the bright variables described here. By adding the pair itself (the two bright $\delta$ Sct stars HD 170782 and HD 170699), that corner of sky looks very promising for a COROT investigation.

\section{Conclusions}

The search for new targets in the COROT accessible field resulted in an exercise of variability detection in stars located in the solar neighbourhood and belonging to the disk population, i.e., in a well-defined galactic region. $\delta$ Sct stars are commonly found; the incidence of this variability is around $25 \%$ of the stars located in the lower part of the instability strip. A similar percentage is observed in the open cluster NGC 6633. The typical pulsator is a multiperiodic one, a little evolved, in the middle part of the strip or slightly cooler. Unfortunately, even if slow rotators are common in the Galaxy, we generally found fast ones $\left(v \sin i>100 \mathrm{~km} \mathrm{~s}^{-1}\right)$ and just one with a very low $v \sin i$, HD 174532. A spectroscopic analysis of the line profile variations will be helpful to estimate the inclination angle $i$ of this promising target.

In the selected sample, 18 stars were located close to or beyond the blue border of the instability strip: in addition to the previously known $\lambda$ Boo star HD 183324, we also found one new $\delta$ Sct star. The observational onset of pulsational instability in this region needs further attention in the future, maybe on the basis of star-by-star investigations.

The results we obtained met the scientific goals of the COROT mission, ensuring a wide choice between $p$-mode pulsators; in general, $\delta$ Sct stars are confirmed to be suitable targets for any asteroseismic space mission, taking into account elemental diffusion and richness of excited modes. Stars appearing as a challenge to theory (HD 170782) or unusual (HD 177702) were also discovered.

Acknowledgements. This research has made use of the SIMBAD database, operating at CDS, Strasbourg, France. The authors wish to thank A. Pamyatnykh and the referee, F. Pijpers, for useful comments, and the STARE team for the attribution of observing time to the COROT project. SM acknowledges financial support from a European Union Marie Curie Fellowship, under contract HPMFCT-2001-01146. RG acknowledges financial support from the program ESP2001-4528-PE. TL, NN and WWW was supported by the Austrian Fonds zur Förderung der wissenschaftliche Forschung 
(P14984) and the BM:WUK (project COROT). PJA acknowledges financial support at the Instituto de Astrofísica de Andalucía-CSIC by an I3P contract (I3P-PC2001-1) funded by the European Social Fund.

\section{References}

Baglin, A., Auvergne, M., Catala, C., et al. 2002, in First Eddington Workshop, Cordoba 11-15 June 2001, ed. J. Christensen-Dalsgaard, \& I. Roxburgh, ESA-SP, 485, 17

Breger, M. 2000, in Delta Scuti and Related Stars, ed. M. Breger, \& M. H. Montgomery, ASP Conf. Ser., 210, 3

Breger, M., \& Pamyatnykh, A. A. 1998, A\&A, 332, 958

Claret, A. 1995, A\&AS, 109, 441

Crawford, D. L., \& Mandwewala, N. 1976, PASP, 88, 917

Guzik, J. A., Bradley, P. A., \& Templeton, M. R. 2000, in Delta Scuti and Related Stars, ed. M. Breger, \& M. H. Montgomery, ASP Conf. Ser., 210, 247

Handler, G. 1999, MNRAS, 309, L19

Handler, G. 2002, in Observational Aspects of Pulsating B and A stars, ed. C. Sterken, \& D. W. Kurtz, ASP Conf. Ser., 256, 113

Handler, G., \& Shobbrook, R. R. 2002, MNRAS, 333, 251
Hildebrandt, G. 1992, AN, 313, 233

Hiltner, W. A., Iriarte, B., \& Johnson, H. L. 1958, ApJ, 127, 539

Martín, S., \& Rodríguez, E. 2002, Eddington Workshop in Cordoba, ESA SP-485, 303

Mermilliod, J.-C. 1995, in Information and On-Line Data in Astronomy, ed. D. Egret, \& M. A. Albrecht (Dordrecht: Kluwer Academic Press), 127

Olsen, E. H. 1993, A\&AS, 102, 89

Pamyatnykh, A. A. 2000, in Delta Scuti and Related Stars, ed. M. Breger, \& M. H. Montgomery, ASP Conf. Ser., 210, 215

Paunzen, E., Handler, G., Weiss, W. W., et al. 2002, A\&A, 392, 515

Philip, A. G. D., \& Egret, D. 1980, A\&A, 40, 199

Philip, A. G. D., Miller, T. M., \& Relyea, L. J. 1976, Dudley Obs. Reports, 12

Poretti, E. 2000, in Delta Scuti and Related Stars, ed. M. Breger, \& M. H. Montgomery, ASP Conf. Ser., 210, 45

Rodríguez, E., \& Breger, M. 2001, A\&A, 366, 178

Sanders, W. L. 1973, A\&AS, 9, 213

Soufi, F., Goupil, M. J., \& Dziembowski, W. A. 1998, A\&A, 334, 911

Templeton, M. R., McNamara, B. J., Guzik, J. A., et al. 1997, AJ, 114, 1592 